\title{
Bemerkungen zu Untersuchungsmethoden und Beurteilung von Nematodenlarven in Wildlachs
}

\author{
Dr. V. Etzel und S. Ramdohr \\ Niedersächsisches Landesamt für Verbraucherschutz und Lebensmittelsicherheit, Cuxhaven (LAVES)
}

\section{Zusammenfassung/Erläuterung}

Zwischen 1999 und April 2006 wurden im IfF Cuxhaven 174 Proben Wildlachs (ganze Fische oder Filets ) untersucht. Bei 138 Proben $(=74 \%)$ waren per enzymatischer Digestion Nematodenlarven ( zwischen 1 und 204 N. - Larven pro kg) nachweisbar. Auffallend scheint eine Zunahme der Nematodenzahl pro Jahr zu sein.

Methodisch wurde verglichen zwischen Durchleuchtung, Bestrahlung mit UV - Licht und Digestion. Die Durchleuchtungsmethode führte mehr oder weniger zufällig zu einem positiven Befund, eine zusätzliche UV - Beleuchtung ließ die Nematodenlarven fluoreszieren, die durch den Filetanschnitt von Fischmuskulatur freigelegt war. Am effektivsten war die anschließende enzymatische Digestion, mit der bei beiden optischen Verfahren keine Nematodenlarven nachweisbar waren.

Bei Wildlachs ist aufgrund seiner zeitweise intensiven Eigenfärbung der Muskulatur die Durch- oder Beleuchtungs- methode nicht geeignet um zu verhindern, dass entsprechend Verordnung ( EG ) Nr. 853/2004 vom 29.4.2004, Kap. V D eindeutig von Parasiten befallene Fischereierzeugnisse für den menschlichen Verzehr in Verkehr gebracht werden können.

Im Rahmen der betrieblichen Eigenkontrollen und zur Überprüfung der in den Betrieben angewandten Sorgfaltspflicht durch die Überwachungsbehörden (VO 854/2004 Anhang III, Kap II, F und Kap. III, Nr. 1) zur Feststellung der Beschaffenheit von Wildlachspartien hinsichtlich der Befallsintensität mit Parasiten ist die Digestionsmethode von hoher Aussagekraft und eigentlich die Methode der Wahl, denn die nach EG-VO 853/2004 vorgeschriebene Durchleuchtungsmethode ist für Wildlachs nicht anwendbar.

\section{Beschluss}

Kenntnisnahme 